\title{
Conceptos actuales sobre el envejecimiento y la enfermedad cardiovascular
}

\author{
Carolina Pemberthy López ${ }^{\mathrm{a}, \mathrm{f}, *}$, Nicolás Jaramillo-Gómez ${ }^{\mathrm{b}, \mathrm{f}}$, \\ Camilo Andrés Velásquez Mejía ${ }^{\mathrm{c}, \mathrm{f}}$, Jonathan Cardona-Vélez ${ }^{\mathrm{d}, \mathrm{f}}$, \\ Heidy Contreras-Martínez ${ }^{e, f}$ y Valentina Jaramillo-Restrepo ${ }^{c, f}$

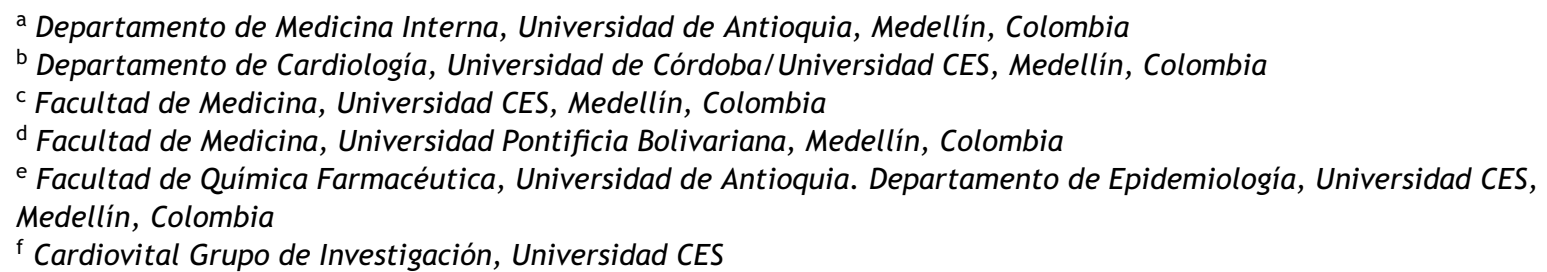

Recibido el 12 de octubre de 2015; aceptado el 14 de diciembre de 2015

Disponible en Internet el 5 de febrero de 2016

\section{PALABRAS CLAVE \\ Enfermedad cardiovascular; \\ Anciano; \\ Enfermedad \\ coronaria; \\ Apoptosis}

\begin{abstract}
Resumen En los últimos años se ha presentado un notable incremento en la proporción de población mayor de 65 años de edad, de la mano con el incremento de las enfermedades cardiovasculares, específicamente la enfermedad coronaria. No obstante, los mecanismos fisiopatológicos del envejecimiento y su relación con la salud cardiovascular son un campo de reciente investigación. Resulta fundamental conocer aspectos como: la teoría del acortamiento de telómeros, el papel de la actividad física, el endotelio y los mecanismos moleculares que participan en este proceso, pues ofrecerá herramientas para hacer un manejo acertado de este grupo poblacional, sobre el cual son pocos los estudios y la mayoría de las conductas son extrapoladas de grupos etarios con condiciones fisiológicas completamente diferentes.

(C) 2016 Sociedad Colombiana de Cardiología y Cirugía Cardiovascular. Publicado por Elsevier España, S.L.U. Este es un artículo Open Access bajo la CC BY-NC-ND licencia (http:// creativecommons.org/licencias/by-nc-nd/4.0/).
\end{abstract}

\section{Current concepts of aging and cardiovascular disease}

Abstract In recent years there has been a marked increase in the proportion of the population over 65 years of age, which has gone hand in hand with the escalation of cardiovascular diseases, particularly coronary disease. However, pathophysiological mechanisms of aging and its relationship to cardiovascular health is a recent field of research. It is fundamental to know aspects

\footnotetext{
* Autor para correspondencia.

Correo electrónico: WEMLcaropember@gmail.com (C. Pemberthy López).
} 
such as the theory of telomere shortening, the role of physical activity, the endothelium and the molecular mechanisms involved in this process, as they will offer tools for a correct management of this population group, upon which few studies are carried out and most behaviours are extrapolated from age groups with completely different physiological conditions.

(c) 2016 Sociedad Colombiana de Cardiología y Cirugía Cardiovascular. Published by Elsevier España, S.L.U. This is an open access article under the CC BY-NC-ND license (http:// creativecommons.org/licenses/by-nc-nd/4.0/).

\section{Introducción}

Con la tendencia demográfica mundial hacia un aumento de la población mayor de 65 años de edad y su implicación en la salud pública, es necesario tener un conocimiento general de la enfermedad cardiovascular en esta franja poblacional, conocer: sus aspectos estructurales, la fisiología y los cambios moleculares, que llevan este grupo etario a ser un blanco más vulnerable de los eventos y secuelas de la enfermedad cardiaca.

Es por este cambio generacional, que se hace necesario emprender un nuevo capítulo en la práctica médica, en donde el adulto mayor sea considerado como un actor principal desde los estudios básicos hasta la transición clínica. Es así, que se decide realizar una revisión de la literatura científica que nos permita elucidar los mecanismos fisiológicos asociados con el envejecimiento cardiovascular, teniendo en cuenta las diferentes teorías que se mueven en el fenómeno del envejecimiento cardiaco para sentar un precedente que lleve a la formulación de posibles elementos de modulación y enlentecimiento de este proceso natural e inevitable.

\section{Epidemiología}

Después del cáncer, las enfermedades cardiovasculares constituyen la segunda causa de mortalidad en el mundo, causando más de 17 millones de muertes. Se presentó un incremento del $7 \%$ de muertes cardiovasculares en todos los grupos etarios entre los años 1990 y 2013. De este número, la enfermedad cardiaca isquémica y la enfermedad cerebrovascular fueron los mayores contribuyentes, reflejándose un aumento del $40 \%$ de mortalidad en la enfermedad isquémica, en donde la enfermedad coronaria fue responsable de 7 millones de muertes ${ }^{1,2}$. La mayor carga de la enfermedad cardiovascular se presenta en países en desarrollo con aproximadamente el $80 \%$ de la totalidad de las muertes por causas cardiovasculares. Los patrones de la enfermedad cardiovascular difieren entre países en desarrollo y países desarrollados, en donde en este último, la mayoría de muertes cardiovasculares se reportan en mayores de 60 años de edad $^{1}$. Según la American Heart Association (AHA), entre los años 2007-2010, la edad promedio de presentación del primer infarto agudo de miocardio fue 64,9 años en los hombres y 72,3 años en las mujeres, con una mortalidad que llega al $80 \%$ de los casos cuando se presenta en mayores de 65 años de edad ${ }^{3}$. La prevalencia del infarto agudo de miocardio aumenta con la edad siendo 7 veces mayor entre los 65-74 años de edad ${ }^{4}$. La población mayor de 65 años de edad comprende en los Estados Unidos 40 millones de individuos, en donde las secuelas cardiovasculares son responsables en gran medida de la mortalidad general en esta población ${ }^{5}$. En Colombia entre los años 1998-2011, las enfermedades cardiovasculares causaron $23,5 \%$ del total de las muertes, de las cuales el $15,2 \%$ se presentaron entre los 65 a 74 años de edad y $37,2 \%$ en $\geq 75$ años de edad; en total el $56,3 \%$ fueron por la enfermedad cardiaca isquémica ${ }^{6}$.

El fenómeno demográfico mundial actual muestra una tendencia de crecimiento desproporcionado de la población añosa. Las Naciones Unidas han calculado que la proporción de la población mayor de 65 años de edad en el mundo se duplicará para el año 2050, estimándose una ocupación del $20 \%$ de la población general ${ }^{5,7}$. Con su implicación económica, elevando hasta un triple los costos de la atención en salud ya que se espera que la enfermedad coronaria y la falla cardiaca aumenten el $200 \%$ para los próximos 20 años ${ }^{8}$.

A pesar de tal crecimiento poblacional, las recomendaciones de manejo en el mundo sobre este grupo poblacional se derivan de un subanálisis de estudios mayores o los análisis retrospectivos de registros con pocas consideraciones específicas para el grupo etario ${ }^{9}$. Los adultos mayores históricamente han tenido poca representación en los estudios clínicos sobre la enfermedad coronaria ${ }^{10}$. Gurwitz y Cols., encontraron que el $60 \%$ de las personas mayores de 75 años de edad, fueron excluidas de los estudios sobre el infarto agudo de miocardio realizados entre los años 1960-1991 ${ }^{11}$. Lee y Cols., reportaron que los mayores de 75 años de edad, fueron excluidos en el $48 \%$ de los estudios publicados entre los años 1991-1995 y en el 32\% de aquellos publicados entre los años 1996 a $2000^{12}$. Por su parte Dodd y Cols., encontraron en una serie de estudios entre los años 2007 a 2009, que sólo el $13.8 \%$ eran mayores de 75 años de edad; de tales estudios el $30 \%$ tenían exclusión de edad, con un límite superior de 80 años en la mayoría ${ }^{13}$. Esta tendencia implica la necesidad de canalizar adecuadamente: los recursos económicos existentes, diseñar estudios que incluyan los pacientes añosos y ampliar los conocimientos científicos sobre el envejecimiento.

El aceptar el reto de ver envejecer la población a una tasa desproporcionada, hace que se deba conocer a una mayor profundidad los fenómenos estructurales, los moleculares y los fisiológicos que la acompañan. Esto nos dará elementos que podrían constituir ayudas para atenuar el impacto social y económico en un futuro inmediato.

\section{Cambios fisiológicos en la vejez}

El envejecimiento según el "nuevo paradigma" se considera como un fenómeno fisiológico determinado y favorecido por la selección supraindividual. Esto implica la existencia 
de mecanismos específicos, genéticamente determinados y regulados que causen el envejecimiento, dentro de los cuales se han descrito la muerte celular programada, el recambio celular, la limitación en la duplicación celular, el acortamiento de los telómeros, entre otros ${ }^{14}$.

\section{Envejecimiento vascular}

En el endotelio, el envejecimiento se asocia con el acortamiento de: los telómeros, la apoptosis, los cambios estructurales y la disfunción de células endoteliales, el incremento de la vasoconstricción dependiente del endotelio, la disminución en la biodisponibilidad del óxido nítrico y la angiogénesis dependiente de endotelio y el aumento de la rigidez vascular mediada por canales endoteliales de sodio $(\text { EnNAC) })^{15,16}$.

Así mismo, se ha determinado que el envejecimiento y las enfermedades crónicas incrementan las propiedades endoteliales "promotoras de la enfermedad" como la endotelina 1, encargada de: estimular la actividad vasoconstrictora, la proliferación celular, la coagulación, la inflamación y la generación de agentes reactivos de oxígeno; así, favorece el desarrollo y la progresión de enfermedades crónicas en relación con la edad avanzada ${ }^{17}$. Frente a ello, los estudios han demostrado que la actividad física regular incrementa la actividad del óxido nítrico, el cual inhibe la endotelina 1 ; ello permite hacer un contrabalance en la progresión del proceso de envejecimiento ${ }^{18}$.

En el músculo liso vascular, el envejecimiento promueve el crecimiento celular, la hipertrofia vascular y el endurecimiento de la pared arterial asociado a la fractura de la elastina en la túnica media y el remodelamiento del colágeno tipo l; en consecuencia incrementa la presión arterial y la presión de pulso con la edad ${ }^{19}$.

El remodelamiento aórtico relacionado con la edad implica un perfil proinflamatorio de las células arteriales y la matriz extracelular. Este perfil implica un aumento de la angiotensina ॥, las moléculas de señalización de receptores de mineralocorticoides y la endotelina, las metaloproteinasas, las proteínas quimiotácticas de monocitos, el factor transformador del crecimiento $\beta 1, T N F-\alpha$, entre otros; se incrementa la activación de TGF- $B$ y NADPH oxidasa en la pared arterial y disminuye la biodisponibilidad del óxido nítrico 20,21 .

Una de las características del proceso de envejecimiento es la presencia de aumento en la sensibilidad endotelial al sodio, dada por un incremento significiativo de los EnNAC con el progresar de los años. Este aumento de receptores endoteliales permite una mayor interacción de sodio-endotelio, generando una respuesta vascular en donde se presenta un incremento en la rigidez de la pared, relacionada con el aumento en el riesgo cardiovascular visto en esta población. Esto, es demostrado en el estudio realizado por Para y Cols. En donde, mediante la evaluación del endotelio y la pared vascular de la aorta en ratones modificados jóvenes y adultos, se encontró un incremento en los receptores EnNAC en la población de mayor edad, y al exponerlos a ambientes hipernatrémicos demostraron un incremento significativo de la rigidez de la pared arterial, el cual fue más marcado en la población adulta. Efecto, que a su vez fue controlado con el uso del amiloride y la espironolactona que se muestran como opciones terapéuticas para la población adulta ${ }^{22}$.

Otros importantes mecanismos implicados en el envejecimiento vascular se describen a continuación:

\section{MFG-E8 (milk fat globule protein epidermal growth factor 8 )}

Se ha descrito la expresión de una proteína denominada MFG-E8 que se incrementa 2-3 veces en la aorta envejecida y se acumula en el contexto del remodelamiento inflamatorio de la pared arterial que ocurre con: la hipertensión, la diabetes mellitus o la aterosclerosis ${ }^{23}$. La proteína MFG-E8 induce la invasión de las células del músculo liso vascular en acción conjunta con la angiotensina ॥ y la proteína quimioatrayente de monocitos 1 , además, estimula su proliferación al coordinar la expresión de moléculas del ciclo celular ${ }^{24}$.

\section{Amiloide}

El amiloide constituye un agregado, producto del mal plegamiento de las proteínas extracelulares que se deposita en los tejidos. La presencia de amiloidosis aórtica es alta a medida que se envejece, la incidencia en estudios realizados con autopsias a nivel de la aorta es hasta de un $79 \%$ en la muestra general, siendo para muestras con edades antes de la quinta década de la vida de $51 \%$, aumentando de forma rápida hasta alcanzar la incidencia del $95 \%$ en la octava década de la vida. La amiloidosis aórtica es mayor que la cardiaca que igualmente aumenta con la edad ${ }^{25}$.

El análisis de proteínas extraídas de una aorta rica en amiloide, detectó unas proteínas amiloide llamada medin. La lactaderina o DEC1 es la precursora del amiloide medin (AMed), que se deposita en la aorta de casi el $100 \%$ de la población caucásica $>50$ años de edad ${ }^{26,27}$. La proteína AMed no se restringe a la aorta y las arterias temporales, también, se encuentra en otras arterias como aquellas de la región superior del cuerpo incluyendo vasos intracraneales ${ }^{28}$. En la aorta de humanos la AMed se deposita junto con las fibras elásticas de las arterias y otras estructuras. In vitro se ha demostrado que esta proteína induce la muerte de las células del músculo liso vascular e induce mayor producción de las metaloproteinasas ${ }^{29}$.

\section{Remodelamiento de matriz extracelular}

Con la edad aumenta la expresión de calpaina-1 y metaliproteinasa 2 en la íntima de la aorta, las cuales participan en dos procesos: 1) La fibrosis de la pared vascular, al incrementar el colágeno tipo । y III; 2) La calcificación de la matriz extracelular, al inducir los factores de crecimiento, degradación de elastina, activación de la fosfatasa alcalina y el contenido total de calcio, sumado a la reducción de inhibidores de calcificación, osteopontina y osteonectina ${ }^{30}$. En estudios en animales el contenido del colágeno aumentó con la edad mientras disminuyó la elastina; ambas reguladas por las metaloproteinasas. Con el envejecimiento, el balance entre las metaloproteinasas y sus inhibidores cambia, presentándose un incremento de las metaloproteinasas a mayor edad ${ }^{31}$. 
Este remodelamiento arterial está relacionado con señales proinflamatorias, incluyendo: el factor de crecimiento transformante $\beta 1$, las proteínas quimioatrayante de monocitos 1 , la proendotelina 1 , activados por las metaloproteinasas de la matriz extracelular ${ }^{32}$.

\section{Calcificación vascular}

La calcificación vascular es un proceso estrechamente regulado, que implica mineralización de la lámina elástica interna y las fibras elásticas en la media, llevando a rigidez arterial. Están implicados: el aumento de los factores de transcripción que regulan la expresión de las proteínas osteogénicas incluyendo la osteocalcina, la osteonectina, la fosfatasa alcalina, el colágeno tipo 1 y la sialoproteína ósea. Otro mecanismo contribuyente es la pérdida de los inhibidores de la calcificación como: la fetuina-A, la proteína de matriz Gla, el pirofosfato y la osteopontina. Evidencia de la relación entre la edad y la calcificación vascular es su presencia en el $5 \%$ de los pacientes menores de 50 años de edad a más de $12 \%$ en aquellos pacientes mayores de 80 años de edad $^{33}$.

\section{Sirtuin (SIRT)}

Es una familia de las deacetilasas y las ribosiltransferasas dependientes de los NAD intracelulares, implicadas en varios procesos celulares asociados con el envejecimiento incluyendo: la apoptosis, la inflamación y la biogénesis mitocondrial. Algunas de sus funciones son: 1) La protección contra la calcificación arterial inducida por fosfato. 2) La regulación de proteínas importantes para la regulación de la función mitocondrial. 3) La potenciación de sistema de defensa antioxidante ${ }^{34}$.

\section{PGC-1 $\alpha$}

La proteína $1 \alpha$ coactivadora del receptor activado por el proliferador de peroxisomas (PGC-1 $\alpha$ ), participa en la regulación de la biogénesis y el recambio mitocondrial, cuya alteración es un importante inductor de los cambios endoteliales relacionados con la edad. En la vasculatura envejecida los niveles reducidos de PGC- $1 \alpha$ conducen a desregulación de la cadena transportadora de electrones llevando a estrés oxidativo y lesión endotelial ${ }^{35}$.

\section{Proteína p66shc}

Es una proteína que participa en la regulación de la producción de especies reactivas del oxígeno mitocondrial y las vías implicadas en la apoptosis y el crecimiento/sobrevida celular. Los niveles de p66shc se incrementan con la edad en: el corazón, el riñón y el músculo liso vascular. Los ratones que carecen del gen p66shc tienen un incremento en la expectativa de vida comparado con los controles salvajes debido a la prevención del estrés oxidativo y la mejoría de la función endotelial ${ }^{36,37}$.

\section{Reguladores del ciclo celular}

Con el envejecimiento celular ocurre la senescencia replicativa en la que la célula queda en fase G1 del ciclo celular y no responde nuevamente a estimulantes de crecimiento. Las células senescentes expresan marcadores específicos ( galactosidasa), sobreexpresan marcadores de ciclo celular (p16 y p21), forman focos de heterocromatina y acumulan lipofuscina, un componente fluorescente no degradable ${ }^{38}$.

Dada la plasticidad de las células de músculo liso vascular, se requiere control estricto en: los procesos transcripcionales, los metabólicos y los ultraestructurales, coordinados a través de la autofagia. La autofagia es un mecanismo que involucra la degradación de las moléculas disfuncionales o innecesarias a través de los lisosomas, jugando un papel crítico en el remodelamiento vascular y en los múltiples procesos relacionados con el envejecimiento y las enfermedades como: la hipertensión arterial y la aterosclerosis ${ }^{29}$.

\section{MAPK (mitogen-activated protein kinase)}

Las proteínas kinasas catalizan la fosforilación de proteínas, cuyos principales blancos son factores de transcripción que modulan las vías de señalización intracelular. Las MAPKs son una familia de proteínas importantes en la transducción de señales en el sistema cardiovascular, implicadas en la regulación de procesos como: la migración celular, la apoptosis, la proliferación, la contracción y la diferenciación. Varios estudios han demostrado un incremento edad dependiente en la activación de MAPK en el tejido vascular ${ }^{24}$.

\section{Estrés oxidativo}

Se ha planteado que la acumulación de radicales libres durante el envejecimiento causa daño de las biomoléculas favoreciendo procesos que promueven: la senescencia celular y el envejecimiento del organismo. La producción excesiva de reactantes de oxígeno lleva a la modificación en las proteínas, el DNA y los lípidos, que se acumulan en las células limitando la función celular y vascular. Además, secundario a la disminución del óxido nítrico, compromete los mecanismos vasodilatadores. El estrés oxidativo participa en varios mecanismos involucrados en el envejecimiento vascular: 1) El aumento de las respuestas proinflamatorias en células vasculares. 2) La disfunción vascular, a través de la modificación de proteínas estructurales y funcionales que regulan: la contracción/relajación vascular, la fibrosis y la calcificación. 3) La alteración de la homeostasis del calcio. 4) La activación de factores de transcripción proinflamatorios y profibróticos. 5) La activación de mecanismos moleculares que llevan a senescencia y autofagia en el endotelio y las células del músculo liso vascular ${ }^{24}$.

A este proceso contribuye la mitocondria, pues con el envejecimiento la mitocondria se vuelve disfuncional, llevando a una reducción en la producción de energía e incremento en la formación de especies reactivas de oxígeno $^{30}$.

Se presentan, además, cambios en el sistema antioxidante que disminuye con en el envejecimiento, a través de 
la regulación a la baja de Nrf2, un factor de transcripción que regula los genes antioxidantes ${ }^{31}$.

\section{Envejecimiento cardiaco}

\section{Teoría del acortamiento de los telómeros}

Los telómeros son secuencias de DNA repetitivas no codificantes ubicadas en ambos extremos de cada cromosoma, donde forman estructuras en asa que evita la fusión con otros cromosomas, protege al DNA de ser reconocido como dañado, reduce el riesgo de la apoptosis y estabiliza el DNA. Durante cada mitosis la DNA polimerasa replica el DNA pero es incapaz de replicar la última parte, llevando a un acortamiento progresivo de los cromosomas. Los extremos de los telómeros se ubican tan cerca del DNA codificante que su función protectora se pierde y el DNA parcialmente dañado es reconocido por supresores de tumores, por los cuales la célula entra en la apoptosis programada o pierde irreversiblemente la habilidad de dividirse, es decir, entra en senescencia replicativa ${ }^{32,33}$.

En un corazón normal cada día mueren 3 millones de miocitos por la apoptosis y son reemplazados por células madres cardiacas, que se duplican y diferencian permitiendo el recambio celular; durante este proceso se presenta progresivamente un acortamiento del telómero relacionado con la edad. En un corazón en su proceso normal de envejecimiento, hay pérdida global de los miocitos por declinación en la habilidad de duplicación de células madre, con aumento compensatorio en el volumen celular de los miocitos restantes, con disminución de la capacidad contráctil cardiaca, agrandamiento cardiaco e hiperplasia de fibroblastos y depósito de colágeno en el intersticio. Ello conduce a la dilatación de las cámaras cardiacas y, a pesar de una atrofia por la disminución en el número de las células, el corazón se torna morfológicamente hipertrófico ${ }^{14}$.

Proceso de envejecimiento basado en nuevos mecanismos moleculares

Los mecanismos moleculares detrás del envejecimiento cardiovascular son pobremente entendidos ${ }^{37}$, por lo que algunas investigaciones han tratado de dar respuesta a través de planteamientos como los siguientes:

\section{ILK- $\beta 1$ integrina}

Las integrinas son receptores de adhesión transmembrana que se unen a la matriz extracelular; su activación afecta la remodelación del citoesqueleto y otras vías de señalización intracelular. En este proceso participan varias proteínas incluidas la ILK (integrin-linked kinase) unida a la $\beta 1$ integrina y a varias proteínas adaptadoras formando una plataforma de señalización. Basados en estudios animales (Drosophila, C. elegans, ratones), se definió la sensibilidad de la ILK, en donde un modelo knock-down moderado de la ILK aumenta la longevidad, frente a una pérdida completa de la ILK que es deletéreo para el desarrollo del organismo. Es interesante, que la sobreexpresión de la ILK en los fibroblastos de ratas induce la senescencia celular y su inhibición la previene. En contraste en los ratones knock-out para ILK se presenta: la dilatación del ventrículo izquierdo, la falla cardiaca, la disgregación del tejido cardiaco y la muerte súbita.
Por tanto, la ILK tiene una función dual: 1) Modula el envejecimiento cardiaco. 2) Mantiene la integridad estructural del corazón ${ }^{37}$.

Estudios recientes en Drosophila demuestran que con una reducción moderada de la señalización integrina/ILK se atenúa el envejecimiento cardiaco normal; se prolonga la expectativa de vida y hay un mejor desempeño cardiaco al envejecer. En contraste, una reducción más severa de la ILK aumenta la incidencia de arritmias y la anormalidad en estructuras cardiacas. Se presenta, además, un aumento de la $\beta 1$ integrina con la edad y su sobreexpresión puede ser deletérea a nivel cardiaco, pudiendo inducir el envejecimiento cardiaco prematuro en moscas jóvenes. De manera similar estudios en monos han determinado la existencia de una importante elevación de $\beta 1$ integrina en células de músculo liso vascular viejas; por lo cual este puede ser un fenómeno común en la vía del envejecimiento miocárdico y vascular $^{37}$.

\section{MicroRNA}

Los MicroRNA son una clase de RNA no codificante que regulan la expresión de múltiples RNAs mensajeros postranscripcionales. Se ha reportado que participan en aspectos cruciales del envejecimiento y la regulación de la función cardiovascular. Por ejemplo, miR-21 y miR-574-5p están incrementados en casos de falla cardiaca y miR-146a y miR-29 durante la senescencia, pero estudios de PCR han demostrado que miR-34a es el que predomina a nivel cardiaco. El silenciamiento in vivo de miR-34a reduce la muerte celular de cardiomiocitos relacionada con la edad. En pacientes con infarto agudo de miocardio (IAM), aumenta su expresión en el borde de la zona del infarto, induciendo fibrosis y muerte celular tanto en cardiomiocitos como en otras células; además, induce senescencia en las células endoteliales e inhibe sus funciones pro-angiogénicas. Así, se ha demostrado que la inhibición de miR-34a disminuye la muerte celular y la fibrosis luego de un IAM y mejora la recuperación de la función cardiaca ${ }^{36}$.

Se han detectado varios blancos del miR-34a, uno de los más estudiados es PNUTS (protein phosphates 1 nuclear targeting subunit o Pp1r10) que es regulado a la baja en corazones envejecidos. Bajo condiciones normales reduce el acortamiento de telómeros, el daño del DNA y la apoptosis de cardiomiocitos y mejora la recuperación funcional después de un IAM; no obstante, estas funciones son suprimidas por la sobreexpresión de miR-34a con la edad. Así, el aumento en la expresión de miR-34a y su acción inhibitoria sobre PNUTS representan un nuevo mecanismo fisiopatológico que explica el detrimento de la función cardiaca durante el envejecimiento e incluso posterior a un evento coronario agudo. Existen estudios sobre otros microRNAs y

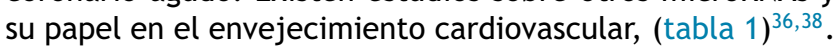

\section{Disminución de células de conducción y endotelio}

Se presenta una disminución progresiva de las células marcapasos del nodo sinusal, asociada a incremento del tejido fibroso, la grasa en el miocardio atrial con disminución en el número de fibras musculares, pérdida de fibras en la rama 
Tabla 1 Función de LncRNA y MicroRNA en el envejecimiento

\begin{tabular}{|c|c|c|c|c|c|}
\hline Genes & Organismo & Órgano/célula & Función & Blanco & Referencia \\
\hline \multicolumn{6}{|l|}{ LncRNAs } \\
\hline Lethe & Ratón & Bazo & Anti-envejecimiento & Rel A & Rapicavoli et al. (2013) \\
\hline XLOC_025918 XLOC_025931 & Humano & Pulmón/fibroblasto & Anti-envejecimiento & No aplica & Abdelmohsen et al. (2013) \\
\hline XLOC_023166 & Humano & Pulmón/fibroblasto & Anti-envejecimiento & No aplica & Abdelmohsen et al. (2013) \\
\hline MALATI & Humano & Pulmón/fibroblasto & Anti-envejecimiento & SRSF1 & Abdelmohsen et al. (2013) \\
\hline MIAT & Humano & Pulmón/fibroblasto & Anti-envejecimiento & No aplica & Abdelmohsen et al. (2013) \\
\hline \multicolumn{6}{|l|}{ MicroRNAs } \\
\hline Lin-4 & C. elegans & No aplica & Anti-envejecimiento & Lin-14 & $\begin{array}{l}\text { Boehm et al. (2005), } \\
\text { Ibanez-Ventoso et al. (2006) }\end{array}$ \\
\hline miR-34 & C. elegans, ratón, humano & $\begin{array}{l}\text { Corazón/cardiomiocito, cél. } \\
\text { endotelial, cél. endotelial }\end{array}$ & Pro-envejecimiento & Sirt1, Pnuts, Atg9a & $\begin{array}{l}\text { Kato et al. (2011), Yang } \\
\text { et al. (2013), Boon et al. }\end{array}$ \\
\hline $\mathrm{miR}-80$ & C. elegans & progenitora & Pro-envejecimiento & $\begin{array}{l}\text { FOXO,hsf-1,cbp-1 } \\
\text { Ctgf, Thbs-1 }\end{array}$ & $\begin{array}{l}\text { (2013), Ito et al. (2010), } \\
\text { Tabuchi et al. (2012), Xu }\end{array}$ \\
\hline miR-17-92-cluster & Ratón & No aplica & Anti-envejecimiento & $\begin{array}{l}\text { Mimecan } \\
\text { Sirt1 }\end{array}$ & $\begin{array}{l}\text { et al. (2012) } \\
\text { Vora et al. (2013) }\end{array}$ \\
\hline miR-22 & Ratón & Corazón/cardiomiocito & Pro-envejecimiento & Nox4 & \\
\hline miR-217 & Humano & & Pro-envejecimiento & & Van Almen et al. (2011) \\
\hline miR-146 & Humano & $\begin{array}{l}\text { Corazón/fibroblasto } \\
\text { Célula endotelial }\end{array}$ & Anti-envejecimiento & Pten, Hmga2 & Jazbutyte et al. (2013) \\
\hline $\operatorname{miR}-21$ & Humano, ratón & Célula endotelial & $\begin{array}{l}\text { Pro-envejecimiento, } \\
\text { Anti-envejecimiento, }\end{array}$ & $\begin{array}{l}\text { Hmga2 } \\
\text { Col1A1, Col3A1, Elastin }\end{array}$ & $\begin{array}{l}\text { Menghini et al. (2009) } \\
\text { Vasa-Nicotera et al. (2011) }\end{array}$ \\
\hline miR-10a & Humano & Cardiomiocito, cél. & Pro-envejecimiento & Atm & Zhang et al. (2012), Rippe \\
\hline miR-29 & Ratón & $\begin{array}{l}\text { endotelial, cél. endotelial } \\
\text { progenitora }\end{array}$ & $\begin{array}{l}\text { Pro-envejecimiento } \\
\text { Pro-envejecimiento }\end{array}$ & & $\begin{array}{l}\text { et al. (2012), Zhu et al. } \\
\text { (2013) }\end{array}$ \\
\hline \multirow[t]{3}{*}{ miR-214 } & Humano & Cél. endotelial progenitora & & & Zhu et al. (2013) \\
\hline & & Aorta & Anti-envejecimiento & & Boon et al. (2011) \\
\hline & & Célula endotelial & & & Burger et al. (2013) \\
\hline
\end{tabular}

LncRNA: Long noncoding RNAs.

Adaptada de Gupta SK, Piccoli MT, Thum T. Non-coding RNAs in cardiovascular ageing. Ageing Res Rev. septiembre de 2014;17:79-85. 
principal del His y en su unión con los fascículos izquierdos, con una menor pérdida en las ramas distales ${ }^{14}$.

El endotelio en su proceso de recambio celular depende de las células progenitoras, cuyo número es inversamente proporcional a la edad y está influenciado de forma negativa por los factores de riesgo cardiovascular ${ }^{14}$. La disfunción endotelial asociada al envejecimiento puede contribuir a la disminución de las respuestas vasodilatadoras coronarias y la angiogénesis ${ }^{35}$, por ello las enfermedades derivadas del compromiso de la función endotelial aumentan exponencialmente con la edad ${ }^{14}$.

Asociado a esto, con el envejecimiento se pierde el preacondicionamiento isquémico, en el cual la exposición a estados isquémicos previos induce la producción de pequeños canalículos colaterales que protegen parcialmente el tejido del miocardio. Ello aumenta la vulnerabilidad de los pacientes mayores a sufrir estados de shock e insuficiencia cardiaca en presencia de un infarto agudo de miocardio ${ }^{32}$.

\section{Actividad física y envejecimiento}

Los cambios morfológicos durante el envejecimiento están determinados también por factores como la actividad física. En estudios realizados en individuos sanos sedentarios, el envejecimiento cardiaco se asoció a una marcada rigidez arterial y cardiaca, que son responsables, en parte de otras enfermedades cardiovasculares como la hipertensión arterial esencial. Además, se han observado cambios como: la acumulación de colágeno, los enlaces cruzados de colágeno en la matriz extracelular, la pérdida de miocitos y el aumento en el tamaño de los miocitos residuales y las alteraciones de la geometría del ventrículo izquierdo (vl), que pueden llevar a alteración de su función diastólica ${ }^{34}$.

Basados en trabajos sobre el envejecimiento saludable en individuos sedentarios, la estructura cardiaca muestra atrofia y un marcado aumento de la rigidez del vı, evaluado por curvas de presión-volumen, lo cual puede ser el sustrato de la falla cardiaca con fracción de eyección preservada (FEp); sin embargo, no está claro a qué edad ocurren estos cambios. Para determinarlo se llevó a cabo un estudio con 70 individuos entre 21-77 años de edad, estratificado por edad, permitiendo identificar diferencias: 1) Un índice de volumen de fin de diástole del vı menor en los $\geq 65$ años. 2) Las curvas de presión-volumen diastólicas del vı mostraron una distensibilidad mayor entre 21-34 años comparado con $\geq 50$ años, que se acentuaba progresivamente con los años. Esto sugiere que la rigidez del vı durante el envejecimiento saludable ocurre durante la transición entre la juventud y la edad media y se hace manifiesta entre los 50-64 años de edad, con el envejecimiento más allá de los 65 años de edad, esta rigidez es seguida por una disminución de la distensibilidad del Vı. Esto permite inferir que la actividad física regular durante la transición entre la juventud y la edad media, puede retardar y atenuar estos cambios ${ }^{34}$.

\section{Conclusiones}

El proceso de envejecimiento reviste una gran cantidad de cambios fisiológicos que determinan un comportamiento particular de la enfermedad cardiovascular. Conocer estos mecanismos abre las puertas a todo un campo de investigación que permita hacer un manejo acertado de la población anciana considerando sus particularidades, pues representa el grupo con mayor crecimiento demográfico y al que el médico se verá enfrentado día a día con mayor frecuencia.

\section{Responsabilidades éticas}

Protección de personas y animales. Los autores declaran que para esta investigación no se han realizado experimentos en seres humanos ni en animales.

Confidencialidad de los datos. Los autores declaran que en este artículo no aparecen datos de pacientes.

Derecho a la privacidad y consentimiento informado. Los autores declaran que en este artículo no aparecen datos de pacientes.

\section{Conflicto de intereses}

Los autores declaran no tener conflictos de intereses.

\section{Bibliografía}

1. Bowry ADK, Lewey J, Dugani SB, Choudhry NK. The Burden of Cardiovascular Disease in Low- and Middle-Income Countries: Epidemiology and Management. Can J Cardiol. 2015;31: 1151-9.

2. Wong ND. Epidemiological studies of $\mathrm{CHD}$ and the evolution of preventive cardiology. Nat Rev Cardiol. 2014;11:276-89.

3. Go AS, Mozaffarian D, Roger VL, Benjamin EJ, Berry JD, Blaha MJ, et al. Heart Disease and Stroke Statistics-2014 Update A Report From the American Heart Association. Circulation. 2014;129:e28-92.

4. Yazdanyar A, Newman AB. The burden of cardiovascular disease in the elderly: morbidity, mortality, and costs. Clin Geriatr Med. 2009;25:563-77, vii.

5. Ahmadi S-F, Streja E, Zahmatkesh G, Streja D, Kashyap M, Moradi $\mathrm{H}$, et al. Reverse Epidemiology of Traditional Cardiovascular Risk Factors in the Geriatric Population. J Am Med Dir Assoc. 2015;16:933-9.

6. Observatorio Nacional de Salud. Enfermedad cardiovascular principal causa de muerte en Colombia. 2013 dic. Report No. 1.

7. Laslett LJ, Alagona P, Clark BA, Drozda JP, Saldivar F, Wilson SR, et al. The Worldwide Environment of Cardiovascular Disease: Prevalence, Diagnosis, Therapy, and Policy Issues. J Am Coll Cardiol. 2012;60:S1-49.

8. Heidenreich PA, Trogdon JG, Khavjou OA, Butler J, Dracup K, Ezekowitz MD, et al. Forecasting the future of cardiovascular disease in the United States: a policy statement from the American Heart Association. Circulation. 2011;123:933-44.

9. Saunderson CED, Brogan RA, Simms AD, Sutton G, Batin PD, Gale CP. Acute coronary syndrome management in older adults: guidelines, temporal changes and challenges. Age Ageing. 2014; 43:450-5.

10. Hanon O, Baixas C, Friocourt P, Carrié D, Emeriau J-P, Galinier $M$, et al. Consensus of the French Society of Gerontology and Geriatrics and the French Society of Cardiology for the management of coronary artery disease in older adults. Arch Cardiovasc Dis. 2009;102:829-45. 
11. Gurwitz JH, Col NF, Avorn J. The exclusion of the elderly and women from clinical trials in acute myocardial infarction. JAMA. 1992;268:1417-22.

12. Lee PY, Alexander KP, Hammill BG, Pasquali SK, Peterson ED. Representation of elderly persons and women in published randomized trials of acute coronary syndromes. JAMA. 2001;286:708-13.

13. Dodd KS, Saczynski JS, Zhao Y, Goldberg RJ, Gurwitz JH. Exclusion of older adults and women from recent trials of acute coronary syndromes. J Am Geriatr Soc. 2011;59: 506-11.

14. Libertini G. The programmed aging paradigm: how we get old. Biochem Biokhimiia. 2014;79:1004-16.

15. Barton M. Aging and endothelin: determinants of disease. Life Sci. 2014;118:97-109.

16. Barton M. Obesity and aging: determinants of endothelial cell dysfunction and atherosclerosis. Pflüg Arch Eur J Physiol. 2010; $460: 825-37$.

17. Wang M, Zhang J, Jiang L-Q, Spinetti G, Pintus G, Monticone R, et al. Proinflammatory profile within the grossly normal aged human aortic wall. Hypertension. 2007;50:219-27.

18. Lakatta EG. The reality of aging viewed from the arterial wall. Artery Res. 2013;7:73-80.

19. Larsson A, Peng S, Persson H, Rosenbloom J, Abrams WR, Wassberg $\mathrm{E}$, et al. Lactadherin binds to elastin-a starting point for medin amyloid formation? Amyloid Int J Exp Clin Investig Off J Int Soc Amyloidosis. 2006;13:78-85.

20. Westermark GT, Westermark P. Localized amyloids important in diseases outside the brain-lessons from the islets of Langerhans and the thoracic aorta. FEBS J. 2011;278:3918-29.

21. Peng S, Glennert J, Westermark P. Medin-amyloid: a recently characterized age-associated arterial amyloid form affects mainly arteries in the upper part of the body. Amyloid Int J Exp Clin Investig Off J Int Soc Amyloidosis. 2005;12:96-102.

22. Paar M, Pavenstadt H, Kusche-Vihrog K, Druppel V, Oberleithner H, Kliche K. Endothelial Sodium Channels Trigger Endothelial Salt Sensitivity With Aging. Hypertension. 2014;64:391-6.

23. Jiang L, Zhang J, Monticone RE, Telljohann R, Wu J, Wang $M$, et al. Calpain-1 regulation of matrix metalloproteinase 2 activity in vascular smooth muscle cells facilitates ageassociated aortic wall calcification and fibrosis. Hypertension. 2012;60:1192-9.

24. Harvey A, Montezano AC, Touyz RM. Vascular biology of ageing-Implications in hypertension. J Mol Cell Cardiol. 2015;83:112-21.
25. Zinovkin RA, Romaschenko VP, Galkin II, Zakharova VV, Pletjushkina OY, Chernyak BV, et al. Role of mitochondrial reactive oxygen species in age-related inflammatory activation of endothelium. Aging. 2014;6:661-74.

26. Lebiedzinska M, Duszynski J, Rizzuto R, Pinton P, Wieckowski MR. Age-related changes in levels of p66Shc and serine 36phosphorylated p66Shc in organs and mouse tissues. Arch Biochem Biophys. 2009;486:73-80.

27. Francia P, Delli Gatti C, Bachschmid M, Martin-Padura I, Savoia C, Migliaccio E, et al. Deletion of p66shc gene protects against age-related endothelial dysfunction. Circulation. 2004;110:2889-95.

28. Lauri A, Pompilio G, Capogrossi MC. The mitochondrial genome in aging and senescence. Ageing Res Rev. 2014;18:1-15.

29. Salabei JK, Hill BG. Autophagic regulation of smooth muscle cell biology. Redox Biol. 2015;4:97-103.

30. Kokoszka JE, Coskun P, Esposito LA, Wallace DC. Increased mitochondrial oxidative stress in the Sod2 (+/-) mouse results in the age-related decline of mitochondrial function culminating in increased apoptosis. Proc Natl Acad Sci U S A. 2001;98:2278-83.

31. Ungvari Z, Bailey-Downs L, Sosnowska D, Gautam T, Koncz P, Losonczy $G$, et al. Vascular oxidative stress in aging: a homeostatic failure due to dysregulation of NRF2-mediated antioxidant response. Am J Physiol Heart Circ Physiol. 2011;301:H363-72.

32. Nilsson PM, Tufvesson H, Leosdottir M, Melander O. Telomeres and cardiovascular disease risk: an update 2013. Transl Res J Lab Clin Med. 2013;162:371-80.

33. Zhang W, Hui R, Yang S. Telomeres, cardiovascular aging, and potential intervention for cellular senescence. Sci China Life Sci. 2014;57:858-62.

34. Fujimoto N, Hastings JL, Bhella PS, Shibata S, Gandhi NK, Carrick-Ranson G, et al. Effect of ageing on left ventricular compliance and distensibility in healthy sedentary humans. J Physiol. 2012;590:1871-80.

35. Majeed F, Kelemen MD. Acute coronary syndromes in the elderly. Clin Geriatr Med. 2007;23:425-40, viii.

36. Boon RA, lekushi K, Lechner S, Seeger T, Fischer A, Heydt $\mathrm{S}$, et al. MicroRNA-34a regulates cardiac ageing and function. Nature. 2013;495:107-10.

37. Nishimura M, Kumsta C, Kaushik G, Diop SB, Ding Y, BisharatKernizan J, et al. A dual role for integrin-linked kinase and $\beta 1$-integrin in modulating cardiac aging. Aging Cell. 2014; 13:431-40.

38. Gupta SK, Piccoli MT, Thum T. Non-coding RNAs in cardiovascular ageing. Ageing Res Rev. 2014;17:79-85. 